\title{
DESIGN AND OPTIMIZATION OF A NOVEL BEAM-BASED SHIMMY DAMPER
}

\author{
Mohsen Rahmani \\ Graduate Student, ARL-MLS Lab \\ MIE Department, University of Toronto \\ Toronto, Canada \\ mohsen.rahmani@mail.utoronto.ca
}

\author{
Kamran Behdinan \\ Professor, ARL-MLS Lab \\ MIE Department, University of Toronto \\ Toronto, Canada \\ behdinan@mie.utoronto.ca
}

\begin{abstract}
Shimmy damper is a passive solution for undesirable oscillations in landing gears. Although it mitigates shimmy to an allowable degree, it can introduce weight, cost, and reliability penalties especially when retrofitted to existing gears. In this report, early investigations into a novel shimmy damper are presented. This damper is suitable for existing and new landing gears and is based on replacing a torque link member with a combination of springs and dampers to suppress the shimmy. The damper is then optimized for a given geometry to deliver the maximum damping force.
\end{abstract}

Keywords- shimmy damper; landing gear; optimization; design;

\section{INTRODUCTION}

Nose Landing Gear (NLG) shimmy is known as selfinduced simultaneous torsional and lateral vibrations arising from the coupling of gear structure, elastic tire(s), and the fuselage structure during ground operations. The oscillations are typically in the range of $10-30 \mathrm{~Hz}$. Shimmy impairs the pilot's visibility and control and causes passenger discomfort, structural damage, as well as sudden failure of the gear [1]. This phenomenon can occur during landing, take-off, and taxying operations. Worn parts contribute to shimmy; however, it also happens in new aircrafts due to the resonance between the gear and the airframe [2]. It is understood that shimmy mode is excited due to transfer of kinetic energy from the moving aircraft to the wheels [3], acting as the energy source for the undesired oscillations. Close to $60 \%$ of aircraft failures are related to the landing gear systems with fatigue due to multi-axial loads (e.g. case of shimmy) playing the number-one role in these accidents [4]. Examples of accidents due to shimmy can be found in [5] and [6]. The latter involved failure of the shimmy damper in addition to the landing gear.

Shimmy needs to be prevented through adequate design during the aircraft development. In practice, shimmy is not discovered until the aircraft is flown and shimmy tests are performed on the ground. In the event of observing shimmy, it is often too late to alter the well-established gear and aircraft design, hence a damper may be designed and prescribed to remedy it. The UTAS (Goodrich) shimmy damper was presented in 2012 [7] which consisted of a damper on one side and a beam with adequate stiffness and strength on the other,

The authors gratefully acknowledge the research grant provided by SPPCA and Natural Sciences and Engineering Research Council of Canada (NSERC) in support of this work. the combination of which makes the shimmy damper. Early studies on the UTAS damper revealed a major issue due to its unsymmetrical design [3]. Although performing better than the flagship Boeing damper [8] in most of the cases, the UTAS damper led to unsymmetrical oscillations which degrades the lifespan of components and tires due to imbalanced loading and uneven wear. The Boeing damper has drawbacks such as adding extra freeplay and weight to the system. Both existing dampers are shown in Fig. 1 along the NLG schematic.
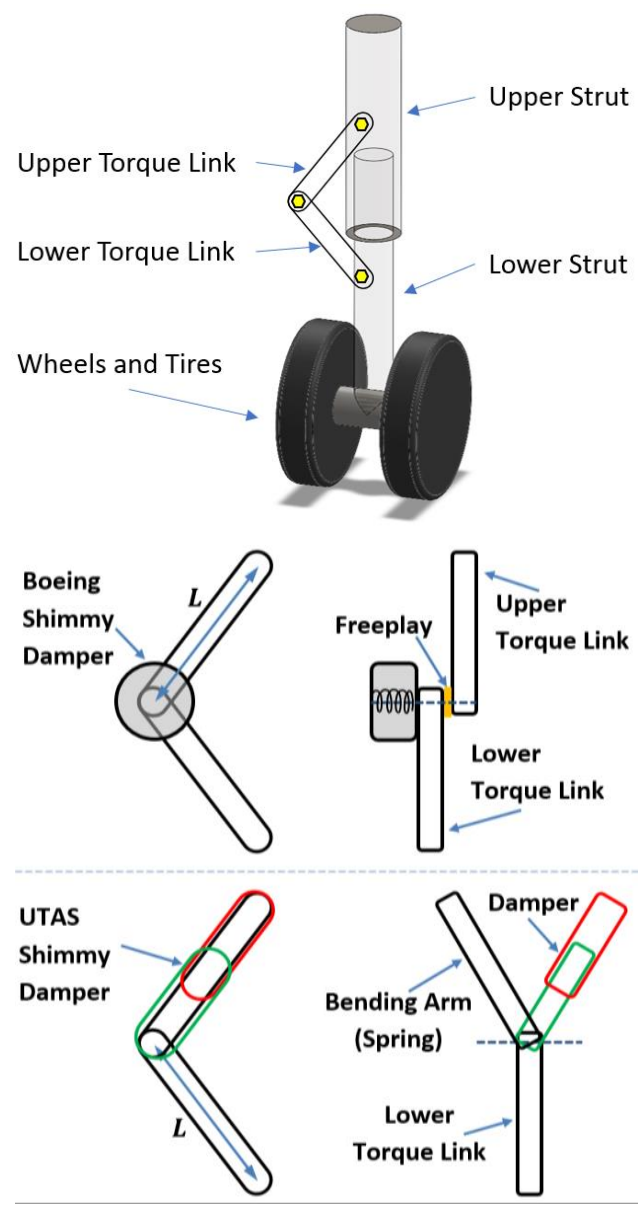

Figure 1. Schematic of the Nose Landing Gear (top), side and front views of Boeing damper (middle) and UTAS damper (bottom) 
Inspired by the UTAS damper and aiming to address its shortcomings, the idea of the present design is to replace one of the torque links (here the upper one) by a flexible beam and accompanying dampers to help stabilize the NLG, while no unsymmetrical behavior is induced in the system.

\section{THE SYMMETRIC BEAM-BASED SHIMMY DAMPER}

As shown in Fig. 2, the upper torque link in the proposed design includes a beam (shaded) at the center and two identical damping devices (here pure dampers) symmetrically arranged on either side. The lower torque link is assumed to be rigid. The beam is the load-bearing element of the upper torque link which acts as a spring in parallel to the side dampers as well.

In the event of shimmy, the lower part of the landing gear (tire and lower strut) tends to vibrate rotationally, hence deflecting the upper torque link. The dampers on either side experience a displacement as a result of the beam deflection. Fig. 3 depicts this effect in which the deflection of the beam at the torque link apex and its rate are designated as $\varepsilon$ and $\dot{\varepsilon}$. The beam deflection and its rate at the damper attachment location are shown as $\varepsilon_{a}$ and $\dot{\varepsilon}_{a}$ respectively. Displacement of the right and left damping devices (measured along the device axis) are $\lambda_{1}$ and $\lambda_{2}$ with rates of $\dot{\lambda}_{1}$ and $\dot{\lambda}_{2}$ respectively.
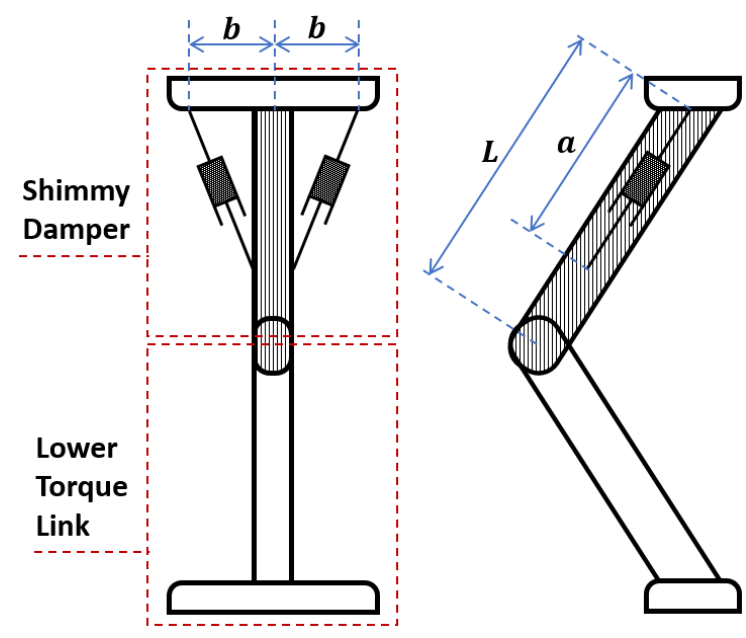

Figure 2. Schematic of the novel shimmy damper and lower torque link. Left: front view, Right: side view

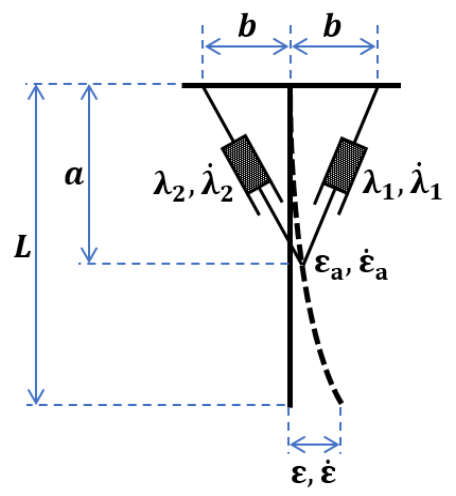

Figure 3. Schematic of the damper beam deflection and damper displacements
Here, the goal is to express the net damping force as a function of the system inputs which are $\varepsilon$ and $\dot{\varepsilon}$. These two inputs are a function of the landing gear dynamics but here are treated as knowns. From the geometry of the design one can write:

$$
\begin{aligned}
& \lambda_{1}=\sqrt{a^{2}+b^{2}-2 b \varepsilon_{a}}-\sqrt{a^{2}+b^{2}} \\
& \lambda_{2}=\sqrt{a^{2}+b^{2}+2 b \varepsilon_{a}}-\sqrt{a^{2}+b^{2}}
\end{aligned}
$$

and hence:

$$
\begin{aligned}
& \dot{\lambda}_{1}=-b \dot{\varepsilon}_{a} / \sqrt{a^{2}+b^{2}-2 b \varepsilon_{a}} \\
& \dot{\lambda}_{2}=b \dot{\varepsilon}_{a} / \sqrt{a^{2}+b^{2}+2 b \varepsilon_{a}}
\end{aligned}
$$

The dampers generate forces along the damper axis which are proportional to $\dot{\lambda}_{1}$ and $\dot{\lambda}_{2}$. However, only the components of these forces which are perpendicular to the beam are going to resist the beam deflection and hence alleviate shimmy. Therefore, we need to isolate the components of $\dot{\lambda}_{1}$ and $\dot{\lambda}_{2}$ which are perpendicular to the beam. These are designated as $\dot{\bar{\lambda}}_{1}$ and $\dot{\bar{\lambda}}_{2}$. To find them we can write:

$$
\begin{gathered}
\bar{\lambda}_{1}=-\lambda_{1}\left(b-\varepsilon_{a}\right) / \sqrt{a^{2}+\left(b-\varepsilon_{a}\right)^{2}} \\
\bar{\lambda}_{2}=\lambda_{2}\left(b+\varepsilon_{a}\right) \sqrt{a^{2}+\left(b+\varepsilon_{a}\right)^{2}} .
\end{gathered}
$$

After taking derivatives and simplifying:

$$
\begin{aligned}
& \dot{\bar{\lambda}}_{1}=-\dot{\lambda}_{1} \frac{b-\varepsilon_{a}}{\sqrt{a^{2}+\left(b-\varepsilon_{a}\right)^{2}}}+\lambda_{1} \frac{a^{2} \dot{\varepsilon}_{a}}{\left(a^{2}+\left(b-\varepsilon_{a}\right)^{2}\right)^{3 / 2}} \\
& \dot{\bar{\lambda}}_{2}=\dot{\lambda}_{2} \frac{b+\varepsilon_{a}}{\sqrt{a^{2}+\left(b+\varepsilon_{a}\right)^{2}}}+\lambda_{2} \frac{a^{2} \dot{\varepsilon}_{a}}{\left(a^{2}+\left(b+\varepsilon_{a}\right)^{2}\right)^{3 / 2}} .
\end{aligned}
$$

Assuming a viscous damper with the coefficient of $C_{d}$ on each side, the total damping force can the expressed as:

$$
Q=C_{d}\left(\dot{\bar{\lambda}}_{1}+\dot{\bar{\lambda}}_{2}\right)
$$


The $L$ and $b$ dimensions are dictated by the geometry and strength of the torque links and existing gear design. However, $a$ needs to be decided by the damper designer in a way to ensure maximum efficiency during the ground operations. Smaller $a$ means the damping devices will be more perpendicular to the beam, but they will experience a smaller beam deflection. The opposite holds for a bigger $a$. Therefore, the optimal value of this parameter need to be determined for the range of inputs generated from the operation of the landing gear, which is the focus of the current study.

It is important to note that the beam deflection is affected by the damper force as well, as depicted in Fig. 4. Hence, the relationship between $\varepsilon$ and $\varepsilon_{a}$ (and their rates) is a function of both $P$ and $Q$ forces, where $P$ is the force applied by the lower torque link. Through governing relationships of a beam with modulus of $E$ and area moment $I$ and assuming small deflections we can write:

$$
\begin{gathered}
E I \varepsilon=\frac{P L^{3}}{3}-\frac{Q a^{2}}{6}(3 L-a) \\
E I \varepsilon_{a}=\frac{P a^{2}}{6}(3 L-a)-\frac{Q a^{3}}{3} .
\end{gathered}
$$

Defining $R_{1}=\varepsilon_{a} / \varepsilon$ and $R_{2}=Q / P$ we will have:

$$
R_{1}=\frac{a^{2}(3 L-a)-2 a^{3} R_{2}}{2 L^{3}-a^{2}(3 L-a) R_{2}} .
$$

Hence, the relationship between the beam deflection at the torque link apex and damper attachment location can be expressed through $\varepsilon_{a}=R_{1} \varepsilon$. For simplicity of the analysis, it is assumed here that the deflection rates are also related through the same ratio, i.e. $\dot{\varepsilon}_{a}=R_{1} \dot{\varepsilon}$, which is to assume that $R_{2}$ does not vary significantly with time. Introducing $R_{1}$ and $R_{2}$ parameters allows us to optimize the performance while considering the effect of the damping force on the beam deflection, and without the need to directly estimate $P$, which is dictated by the NLG dynamics.

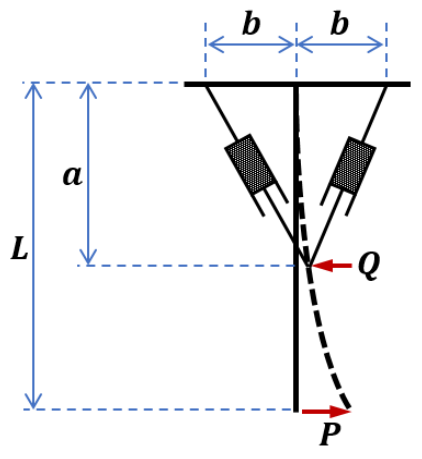

Figure 4. The forces applied to the beam

\section{PERFORMANCE ANALYSIS}

The analysis is commenced by looking at the simpler case when the influence of damping force on the beam deflection is neglected, i.e. $R_{2}=0$. Later, we quantify the effect of this simplifying assumption. The geometry features of $L=0.4 \mathrm{~m}$ and $b=0.25 L$ are assumed as our demonstration case and will be used throughout the paper unless otherwise stated. The net damping force is depicted as a function of $a / L$ in Fig. 5 with the optimal location (corresponding to the maximum damping force) marked as a star on each curve. For all cases that follow, unit damper coefficient and apex displacement rate $\left(C_{d}=1\right.$, $\dot{\varepsilon}=1$ ) are used since the net damping force is a linear function of these parameters. From Fig. 5 one observes that the optimal damper location is a function of $\varepsilon$. However, for the practical range of $0 \leq \varepsilon \leq 0.05 \mathrm{~m}$ the optimal $a / L$ only changes from 0.63 to 0.58 . Therefore, one may choose $a / L \approx 0.6$ for this case as a value which provides near-optimal performance for the intended range of operation.

Next, the effect of $b$ parameter is demonstrated for a fixed apex deflection of $\varepsilon=0.01 \mathrm{~m}$. Again, $R_{2}=0$ and damping force is shown in Fig. 6. Typically, $b$ is not a design variable since it is dictated by the design of the gear to which the shimmy damper will be retrofitted. Nevertheless, it is beneficial to understand that a bigger $b$ leads to a bigger optimal $a / L$ and a larger damping force as shown in Fig. 6.

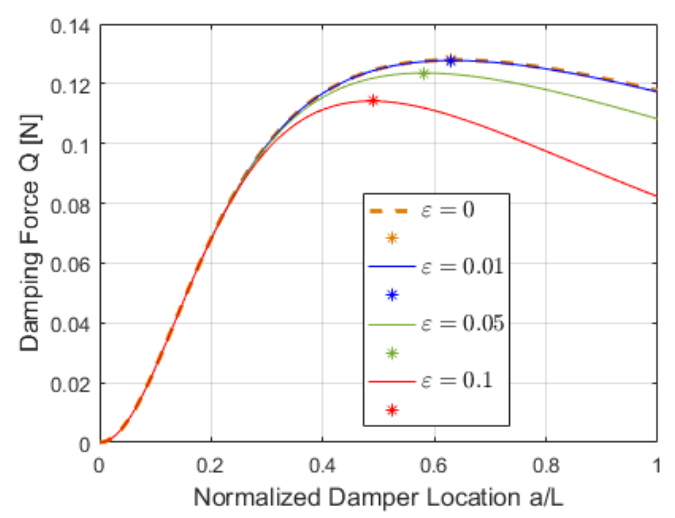

Figure 5. Damping force versus damper location for $R_{2}=0$ and various $\varepsilon$

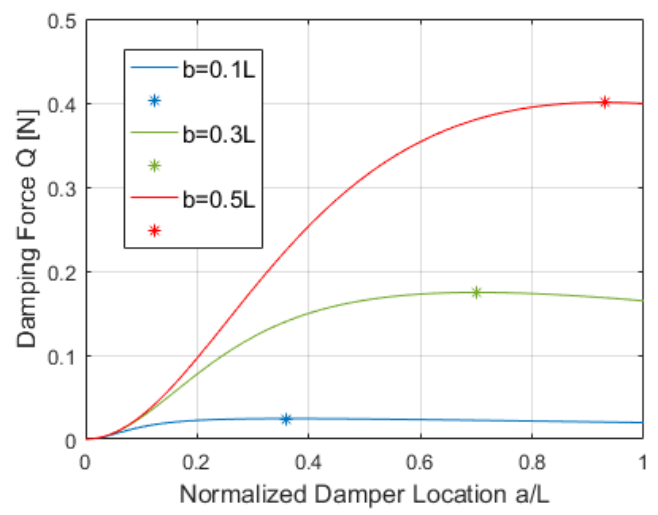

Figure 6. Damping force versus damper location for $R_{2}=0$ and various $b$ 


\section{A. Effect of $R_{2}=0$ Assumption}

To account for the effect of damping force on the beam deflection we need to understand the influence of $R_{2}$ parameter on the predicted response. In practice, this value is non-zero due to presence of the damping force $Q$. In fact, we are dealing with a loop here: the calculation of damping force needs the accurate beam deformation and the beam deformation can be obtained by knowing the damping force. Hence, one may go through an iterative scheme to get the solution. Instead, here we calculate the solution for different values of $R_{2}$ parameter which indicates how sensitive to the damping force the exact solution is.

In Fig. 7, the damping force and optimal damper location are depicted for various values of $R_{2}$ for both small $(\varepsilon=0.01 \mathrm{~m})$ and large $(\varepsilon=0.1 \mathrm{~m})$ apex displacement values. As expected, it is observed that a non-zero $R_{2}$ alters the estimated damping force curve and the optimal damper location. Hence, the optimal damper location is underpredicted when assuming $R_{2}=0$. However, for small values of $R_{2}$ the difference is negligible, and one can proceed with the $R_{2}=0$ assumption for initial concept design studies. Furthermore, the influence of $R_{2}$ on the optimal damper location decreases as $\varepsilon$ grows. This is key since performance at larger $\varepsilon$ values should drive the design as it corresponds to severe shimmy situations.
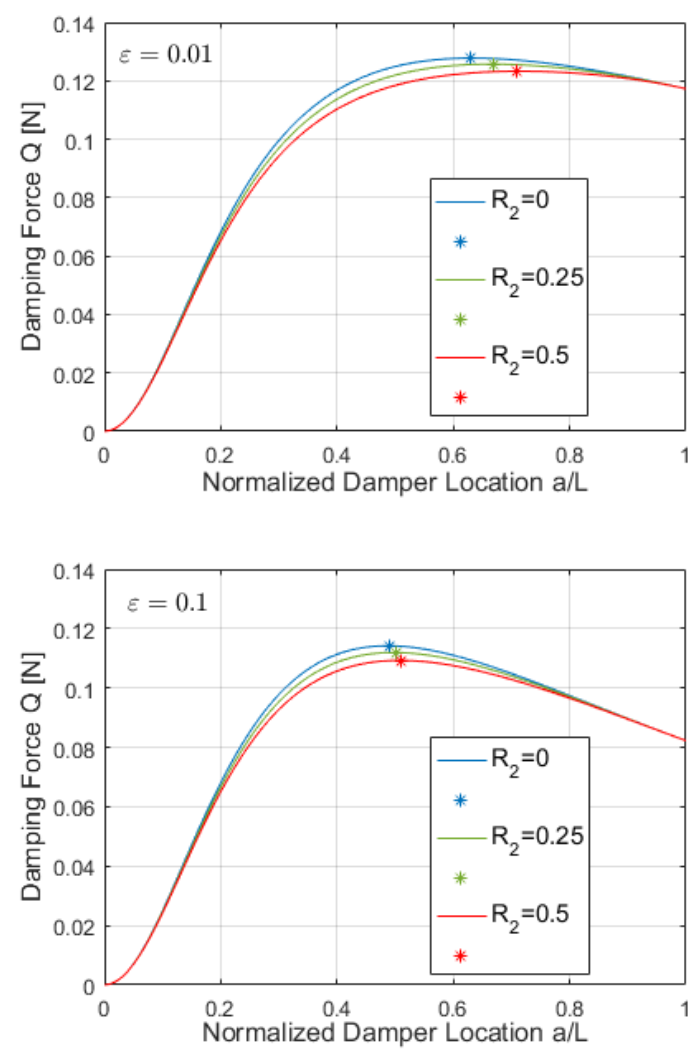

Figure 7. Damping force versus damper location for various $R_{2}$. Top: $\varepsilon=0.01 \mathrm{~m}$, bottom: $\varepsilon=0.1 \mathrm{~m}$

\section{OPTIMIZATION}

Using the analyses presented so far, we can arrive at a more complete picture of the optimal damper location for a given geometry and range of operation. Here the torque link dimensions are given as $L=0.4 \mathrm{~m}$ and $b=0.25 L$. The torque link apex displacement $\varepsilon$ is assumed to be in the $0 \leq \varepsilon \leq 0.1 \mathrm{~m}$ range. Fig. 8-top shows the optimal location versus $\varepsilon$ for different values of $R_{2}$. Assuming $R_{2}=0$ results in a smaller $a_{\mathrm{opt}} / L$ but the error can be acceptable considering the curves are converging as $\varepsilon$ grows. For instance, in the case of a given shimmy-prone landing gear with maximum apex displacement of $\varepsilon=0.04 \mathrm{~m}$ one can choose $a_{\mathrm{opt}} / L=0.6$ (assuming $R_{2}=0$ ) according to Fig. 8-top to ensure the damper performance is optimal for the maximum $\varepsilon$ and near optimal (since the lines are not steep) for smaller values. Similar curves are plotted for different $b$ values in Fig. 8-bottom. The predicted trend is that the $a_{\text {opt }} / L$ is a function of $\varepsilon$ but may be fixed at the value corresponding to the maximum $\varepsilon$ in the operation range to ensure effective shimmy suppression. This embodies a worstcase scenario design strategy where the performance of the system is optimal for the severe shimmy case and near optimal for other cases. Although this decision is adequate for the initial concept design, one needs to perform more thorough analysis involving real inputs from the NLG to ensure shimmy is suppressed effectively.
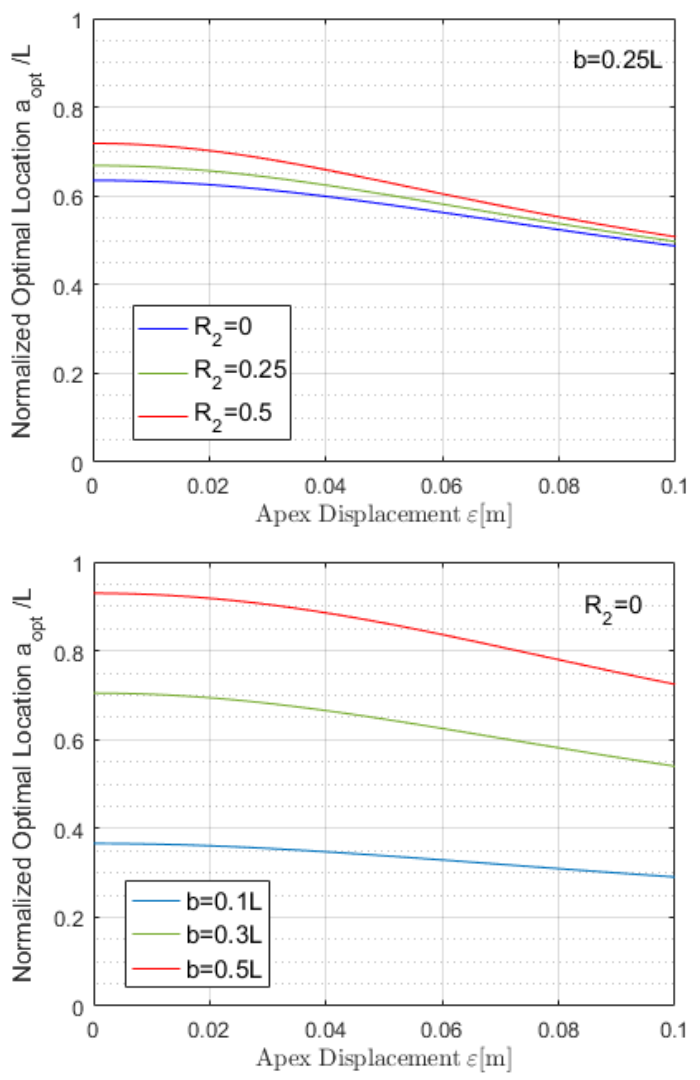

Figure 8. Optimal damper location versus $\varepsilon$ for various $R_{2}$ values (top) and various $b$ values (bottom) 


\section{CONCLUSIONS}

A novel shimmy damper concept is featured in the current work and initial optimization studies are presented to ensure maximum efficiency of the damper for the most critical shimmy scenario. This damper is superior to the Boeing one in that it does not add freeplay and is easily retrofitted to existing gears. It also addresses the unsymmetrical response issue of the UTAS damper. The evidence of symmetric response of the damper based on dynamic simulation of the NLG equipped with the damper remains to be obtained, although the symmetric design of the device promises such performance.

The detail design of the beam and damping devices, as well as multi-disciplinary optimization studies require a computational dynamic model of the NLG such as [9] and [10]. The exact damping force can be estimated by adding the present damper model to the landing gear dynamic model which can provide the inputs in real-time. The damping force should also be fed back to the NLG system and the performance of the damper needs to be evaluated in ground operation simulations such as a landing scenario. Furthermore, more advanced combinations of springs, dampers, and inerters will be considered as damping devices to arrive at a more efficient and compact design. These ideas are under investigation by the authors and comprise the next steps of the present work.

\section{REFERENCES}

[1] W. Krüger, I. Besselink, D. Cowling, D. Doan, W. Kortüm and W. Krabacher, "Aircraft landing gear dynamics: simulation and control," Veh. Syst. Dyn., vol. 28, no. 2-3, pp. 119-158, 1997.

[2] W. E. Luce, "Torque link with shimmy damper", U.S. Patent No. U.S. 8448899 B2, 2013

[3] C. Arreaza, K. Behdinan and J. Zu, "Linear stability analysis and dynamic response of shimmy dampers for main landing gears", J. Appl.Mech., vol. 83, no. 8, pp. 081002-081002-10, 2016.

[4] S. Lok, J. Paul, and V. Upendranath, "Prescience life of landing gear using multiaxial fatigue numerical analysis" Procedia Eng., vol. 86, pp. 775-779, 2014

[5] P. Eret, J. Kennedy, and G. Bennett, "Effect of noise reducing components on nose landing gear stability for a mid-size aircraft coupled with vortex shedding and freeplay", J. Sound Vib., vol. 354, pp. 91-103, 2015.

[6] Accident of aircraft Fokker MK-100, registration I-ALPL, at Barcelona Airport (Barcelona), on 7 November 1999. Technical Report A068/1999, 1999.

[7] W. E. Luce, "Torque link with shimmy damper", U.S. Patent No. U.S. 8292218 B2, 2012.

[8] W. E. Boehringer, "Torque linkage damper" U.S. Patent No. 5224668, 1993.

[9] M. Rahmani, K. Behdinan, "Investigation on the effect of coulomb friction on nose landing gear shimmy", submitted to J. Vib. Control.

[10] P. Thota, B. Krauskopf and M. Lowenberg, "Bifurcation analysis of nose-landing gear shimmy with lateral and longitudinal bending", J. Aircraft, vol. 47, no.1, pp. 87-95, 2010. 